BULLETIN (New Series) OF THE AMERICAN MATHEMATICAL SOCIETY

Volume 35, Number 3, July 1998, Pages 215-230

S 0273-0979(98)00755-1

\title{
NORMAL FAMILIES: NEW PERSPECTIVES
}

\author{
LAWRENCE ZALCMAN
}

\begin{abstract}
This paper surveys some surprising applications of a lemma characterizing normal families of meromorphic functions on plane domains. These include short and efficient proofs of generalizations of (i) the Picard Theorems, (ii) Gol'dberg's Theorem (a meromorphic function on $\mathbb{C}$ which is the solution of a first-order algebraic differential equation has finite order), and (iii) the Fatou-Julia Theorem (the Julia set of a rational function of degree $d \geq 2$ is the closure of the repelling periodic points). We also discuss Bloch's Principle and provide simple solutions to some problems of Hayman connected with this principle.
\end{abstract}

Over twenty years ago, on the way to a partial explication of the phenomenon known as Bloch's Principle, I proved a little lemma characterizing normal families of holomorphic and meromorphic functions on plane domains [68]. Over the years, the lemma has grown and, in dextrous hands, proved amazingly versatile, with applications to a wide variety of topics in function theory and related areas. With the renewed interest in normal families ${ }^{1}$ (arising largely from the important role they play in complex dynamics), it seems sensible to survey some of the most striking of these applications to the one-variable theory, with the aim of making this technique available to as broad an audience as possible. That is the purpose of this report.

One pleasant aspect of the theory is that judicious application of the lemma often leads to proofs which seem almost magical in their brevity. In such cases, we have made no effort to resist the temptation to write out complete proofs. Hardly anything beyond a basic knowledge of function theory is required to understand what follows, so the reader is urged to take courage and plough on through. And now we turn to our tale.

1. Let $D$ be a domain in the complex plane $\mathbb{C}$. We shall be concerned with analytic maps (i.e., meromorphic functions)

$$
f:\left(D,|\quad| \mathbb{R}^{2}\right) \rightarrow(\hat{\mathbb{C}}, \chi)
$$

Received by the editors October 15, 1997, and, in revised form, May 26, 1998.

1991 Mathematics Subject Classification. Primary 30D45; Secondary 30D35, 34A20, 58F23.

Key words and phrases. Normal families, Picard's Theorem, algebraic differential equations, Julia set, Bloch's Principle.

${ }^{1}$ Witnessed by the appearance in recent years of not one, but two treatises on the subject [15], [59] — after a hiatus of over sixty years!

(C) 1998 American Mathematical Society 
from $D$ (endowed with the Euclidean metric) to the extended complex plane $\hat{\mathbb{C}}$, endowed with the chordal metric $\chi$, given by

$$
\begin{aligned}
\chi\left(z, z^{\prime}\right) & =\frac{\left|z-z^{\prime}\right|}{\sqrt{1+|z|^{2}} \sqrt{1+\left|z^{\prime}\right|^{2}}} \quad z, z^{\prime} \in \mathbb{C} \\
\chi(z, \infty) & =\frac{1}{\sqrt{1+|z|^{2}}} .
\end{aligned}
$$

A family $\mathcal{F}$ of meromorphic functions on $D$ is said to be normal on $D$ if each sequence $\left\{f_{n}\right\} \subset \mathcal{F}$ has a subsequence which converges $\chi$-uniformly on compact subsets of $D$. It is easy to see that in case all functions in $\mathcal{F}$ are holomorphic, this condition is equivalent to the requirement that each sequence $\left\{f_{n}\right\} \subset \mathcal{F}$ have a subsequence which either converges uniformly (with respect to the Euclidean metric) on compacta in $D$ or diverges uniformly to $\infty$ on compacta in $D$.

Normality is, quite clearly, a compactness notion: a family $\mathcal{F}$ of meromorphic functions on $D$ is normal if and only if it is precompact in the topology of $\chi$-uniform convergence on compact subsets of $D$. Accordingly, by the Arzela-Ascoli Theorem, normality is equivalent to equicontinuity on compacta of the functions in $\mathcal{F}$. Since these are smooth functions, this equicontinuity should be equivalent to the local boundedness of an appropriate derivative. Such is the content of

Marty's Theorem. [38] A family $\mathcal{F}$ of functions meromorphic on $D$ is normal on $D$ if and only if for each compact subset $K \subset D$ there exists a constant $M(K)$ such that

$$
f^{\#}(z) \leq M(K)
$$

for all $z \in K$ and all $f \in \mathcal{F}$.

Here $f^{\#}$ denotes the spherical derivative

$$
\begin{array}{rlrl}
f^{\#}(z) & =\lim _{h \rightarrow 0} \frac{\chi(f(z+h), f(z))}{|h|} & \\
& =\frac{\left|f^{\prime}(z)\right|}{1+|f(z)|^{2}} \quad(f(z) \neq \infty) .
\end{array}
$$

Since $\chi(z, w)=\chi(1 / z, 1 / w), f^{\#}=(1 / f)^{\#}$, which provides a convenient formula for $f^{\#}$ at poles of $f$.

Armed with this background, we can now engage the subject of our survey.

2. In its most general form, the result we shall be concerned with may be stated as follows.

Lemma. Let $\mathcal{F}$ be a family of meromorphic functions on the unit disc $\Delta$ such that all zeros of functions in $\mathcal{F}$ have multiplicity greater than or equal to $\ell$ and all poles of functions in $f$ have multiplicity greater than or equal to $j$. Let $\alpha$ be a real number satisfying $-\ell<\alpha<j$. Then $\mathcal{F}$ is not normal in any neighborhood of $z_{0} \in \Delta$ if and only if there exist

(i) points $z_{k} \in \Delta, z_{k} \rightarrow z_{0}$;

(ii) positive numbers $\rho_{k}, \rho_{k} \rightarrow 0$; and

(iii) functions $f_{k} \in \mathcal{F}$ 
such that $\rho_{k}^{\alpha} f_{k}\left(z_{k}+\rho_{k} \zeta\right) \rightarrow g(\zeta)$ spherically uniformly on compact subsets of $\mathbb{C}$, where $g$ is a nonconstant meromorphic function. The function $g$ may be taken to satisfy the normalization

$$
g^{\#}(z) \leq g^{\#}(0)=1 \quad z \in \mathbb{C} .
$$

This is Pang's generalization [48], [49] (cf. [65]) of the Main Lemma in [68] (where $\alpha$ is taken to be 0), with improvements due to Schwick [60] and Chen and $\mathrm{Gu}$ [13]; cf. [51].

By way of illustration, consider the non-normal family $\mathcal{F}=\left\{(2 z)^{k}\right\}$ on $\Delta$. Choosing $\alpha=0, f_{k}(z)=(2 z)^{k}, z_{k}=1 / 2$, and $\rho_{k}=a / 2 k$, we have $f_{k}\left(z_{k}+\rho_{k} \zeta\right)=$ $(1+a \zeta / k)^{k} \rightarrow e^{a \zeta}$ on $\mathbb{C}$. For the normalization $g^{\#}(z) \leq g^{\#}(0)=1$, choose $a=2$.

Clearly, with no special restrictions on the zeros and poles of functions in $\mathcal{F}$, the Lemma holds for $-1<\alpha<1$; on the other hand, if all functions in $\mathcal{F}$ are holomorphic (so that the condition on the poles is satisfied vacuously for arbitrary $j$ ), we may take $-1<\alpha<\infty$. Similarly, for families of meromorphic functions which do not vanish, one may choose $-\infty<\alpha<1$. In point of fact, for most applications the choice $\alpha=0$ is sufficient. However, for dealing with families of functions defined by conditions on derivatives, it is essential to be able to choose $\alpha$ appropriately; cf. $\S 8$.

A word is in order concerning the condition that the limit function $g$ have bounded spherical derivative. This requirement imposes severe restrictions on the growth of $g$; specifically $g$ must be of finite order. Recall that the order of a meromorphic function $g$ on the plane is given by

$$
\rho=\varlimsup_{r \rightarrow \infty} \frac{\log T_{0}(r)}{\log r} .
$$

Here $T_{0}(r)$ is the Ahlfors-Shimizu characteristic of $g$, defined by

$$
T_{0}(r)=\int_{0}^{r} \frac{S(t)}{t} d t
$$

where

$$
S(t)=\frac{1}{\pi} \iint_{|z| \leq t}\left[g^{\#}(z)\right]^{2} d x d y
$$

is the (normalized) area on the sphere of $g(\{|z| \leq t\}$ ), counting multiplicities. In particular, if $g^{\#}$ is bounded, then $T_{0}(r)=O\left(r^{2}\right)$ so that $\rho \leq 2$. For holomorphic functions, even more is true: bounded spherical derivative implies exponential type [17], cf. [39]. In general, the limit functions which arise by the process described in the lemma need not have bounded spherical derivative. The possibility of choosing a function satisfying this additional condition plays an important role in certain applications; cf. $\S \S 3,8$.

Let us sketch the proof of the Lemma in the case $\alpha=0$. Suppose first that $\mathcal{F}$ is normal on $\Delta$ and that (i)-(iii) hold. Choose $r$ such that $\left|z_{k}\right| \leq r<1$. By Marty's Theorem, there exists $M>0$ such that

$$
\max _{|z| \leq(1+r) / 2} f^{\#}(z) \leq M
$$

for all $f \in \mathcal{F}$. Fix $\zeta \in \mathbb{C}$. For large $k,\left|z_{k}+\rho_{k} \zeta\right| \leq(1+r) / 2$, so that $\rho_{k} f_{k}^{\#}\left(z_{k}+\rho_{k} \zeta\right) \leq$ $\rho_{k} M$. Thus, for all $\zeta \in \mathbb{C}, g^{\#}(\zeta)=\lim \rho_{k} f_{k}^{\#}\left(z_{k}+\rho_{k} \zeta\right)=0$. It follows that $g$ is a constant (possibly infinity). 
Conversely, if $\mathcal{F}$ is not normal on $\Delta$, by Marty's Theorem there exists a number $r^{*}, 0<r^{*}<1$, points $z_{k}^{*}$ in $\left\{|z| \leq r^{*}\right\}$, and functions $f_{k} \in \mathcal{F}$ such that $f_{k}^{\#}\left(z_{k}^{*}\right) \rightarrow$ $\infty$. Fix $r, r^{*}<r<1$, and let

$$
M_{k}=\max _{|z| \leq r}\left(1-\frac{|z|^{2}}{r^{2}}\right) f_{k}^{\#}(z)=\left(1-\frac{\left|z_{k}\right|^{2}}{r^{2}}\right) f_{k}^{\#}\left(z_{k}\right) .
$$

The maximum exists since $f_{k}^{\#}$ is continuous for $|z| \leq r$. Clearly we have $M_{k} \geq$ $\left(1-\left|z_{k}^{*}\right|^{2} / r^{2}\right) f_{k}^{\#}\left(z_{k}^{*}\right) \rightarrow \infty$. Put

$$
\rho_{k}=\frac{1}{M_{k}}\left(1-\frac{\left|z_{k}\right|^{2}}{r^{2}}\right)=\frac{1}{f_{k}^{\#}\left(z_{k}\right)} .
$$

This works!

(In case $z_{0}$ is the only point in $\Delta$ at which $\mathcal{F}$ fails to be normal, it is automatic that the sequence $\left\{z_{n}\right\}$ chosen above converges to $z_{0}$. Otherwise, a slightly different argument, amounting to a diagonalization of the previous reasoning, is needed to insure that $z_{k} \rightarrow z_{0}$; cf. [60] pp. 242-244.)

3. A central result in the theory of normal families is Montel's Theorem, according to which a family of functions meromorphic on a domain $D$, all of which fail to take on three fixed (and distinct) values in $\widehat{\mathbb{C}}$, is normal on $D$. It is this theorem, which Schiff calls the Fundamental Normality Test ([59] pp. 54,74), that makes available the mechanism of normal families for proving global results in (one-dimensional) complex dynamics [21] p. 9. Here is a simple and elementary proof of Montel's Theorem, based on an idea of Antonio Ros [55].

Montel's Theorem. The collection $\mathcal{F}$ of all meromorphic functions which omit three fixed values $a, b, c \in \hat{\mathbb{C}}$ on a domain $D \subset \mathbb{C}$ is a normal family on $D$.

Proof. Since normality is a local notion, we may suppose that $D=\Delta$, the unit disc. Composing with a linear fractional transformation, we may also assume that the omitted values are $0,1, \infty$. Let us denote by $\mathcal{F}_{n}$ the collection of functions on $\Delta$ which omit the values $0, \infty$, and all $n$th roots of 1 , so that $\mathcal{F}=\mathcal{F}_{1}$. Note that $f \in \mathcal{F}$ implies $\sqrt[n]{f} \in \mathcal{F}_{n}$, while if $h \in \mathcal{F}_{n}$, then $h^{n} \in \mathcal{F}$.

Suppose now that $\mathcal{F}$ is not normal. Then none of the families $\mathcal{F}_{n}$ is normal, so by the Lemma we have, for each $n$, a nonconstant entire function $g_{n}$ obtained as a limit of functions omitting all values in $S_{n}=\left\{0,1, e^{2 \pi i k / n}, k=0,1, \ldots, n-1\right\}$. By Hurwitz's Theorem, $g_{n}$ also omits $S_{n}$. Moreover, $g_{n}^{\#}(z) \leq g_{n}^{\#}(0)=1$.

Write, for convenience, $T_{n}=S_{2^{n}}, G_{n}=g_{2^{n}}$, and consider the family $\mathcal{G}=\left\{G_{n}\right\}$ on $\mathbb{C}$. Now $G_{n}^{\#}(z) \leq 1$ for all $z \in \mathbb{C}$, so by Marty's Theorem $\mathcal{G}$ is normal on $\mathbb{C}$; hence a subsequence converges, $\chi$-uniformly on compacta, to a limit function $G$. Since $G_{n}^{\#}(0)=1$ for all $n, G^{\#}(0)=1$, so $G$ is nonconstant. The sets $T_{n}$ are nested, so that $G_{m}$ omits values in $T_{n}$ as soon as $m \geq n$. By Hurwitz's Theorem, $G$ must omit $T_{n}$ for every $n$. Since $\cup T_{n}$ is dense in the unit circle and $G(\mathbb{C})$ is an open connected set, this implies that either $G(\mathbb{C}) \subset \Delta$ or $G(\mathbb{C}) \subset \mathbb{C} \backslash \bar{\Delta}$. In either case, we have a contradiction to Liouville's Theorem.

Immediate (and easy) corollaries of Montel's Theorem include the theorems of Picard, as well as the existence of a direction of Julia for entire functions [58] p. 352. The proof just given, together with the standard deduction of Picard's Great Theorem from Montel's Theorem ([58] p. 351), provides what is to my mind the shortest and simplest route to this pinnacle of complex function theory. 
Montel's Theorem itself admits various generalizations ([59] pp. 104-105), some of which are mentioned in $\S 7$ below.

4. According to Marty's Theorem, a family $\mathcal{F}$ of meromorphic functions is normal on $D \subset \mathbb{C}$ if and only if for each compact set $K \subset D$ there exists a constant $M(K)$ such that $f^{\#}(z) \leq M(K)$ for all $f \in \mathcal{F}$ and all $z \in K$. The following lovely application of the Lemma allows us to reduce drastically the set on which this inequality is required to hold.

Theorem. (Lappan [36]; cf. [35], [14], [30]) A family $\mathcal{F}$ of meromorphic functions is normal on $D \subset \mathbb{C}$ if and only if for each compact set $K \subset D$ there exists a set $E=E(K) \subset \hat{\mathbb{C}}$ containing at least five points and a constant $M=M(K)>0$ such that

$$
f^{\#}(z) \leq M \quad z \in K, \quad f(z) \in E
$$

for all $f \in \mathcal{F}$.

Proof. Marty's Theorem shows that (3) is necessary with $E=\hat{\mathbb{C}}$. To prove sufficiency, suppose that (3) holds but $\mathcal{F}$ is not normal. Then we can find $f_{k} \in \mathcal{F}$, $z_{k} \rightarrow z_{0} \in D$, and $\rho_{k} \rightarrow 0+$ such that $f_{k}\left(z_{k}+\rho_{k} \zeta\right)=g_{k}(\zeta) \rightarrow g(\zeta)$ spherically uniformly on compacta, where $g$ is a nonconstant meromorphic function on $\mathbb{C}$. Let $K \subset D$ be a closed disc about $z_{0}$ and suppose $g\left(\zeta_{0}\right) \in E$. By Hurwitz's Theorem, there exist $\zeta_{k} \rightarrow \zeta_{0}$ such that $f_{k}\left(z_{k}+\rho_{k} \zeta_{k}\right)=g_{k}\left(\zeta_{k}\right)=g\left(\zeta_{0}\right)$ for large $k$. Now by (3), $f_{k}^{\#}\left(z_{k}+\rho_{k} \zeta_{k}\right) \leq M$ for $k$ sufficiently large, so that

$$
g^{\#}\left(\zeta_{0}\right)=\lim _{k \rightarrow \infty} g_{k}^{\#}\left(\zeta_{k}\right)=\lim _{k \rightarrow \infty} \rho_{k} f_{k}^{\#}\left(z_{k}+\rho_{k} \zeta_{k}\right) \leq \lim _{k \rightarrow \infty} \rho_{k} M=0 .
$$

Thus $g^{\#}\left(\zeta_{0}\right)=0$ whenever $g\left(\zeta_{0}\right) \in E$, i.e., each value in $E$ is totally ramified. But it is a well-known consequence of Nevanlinna's Second Fundamental Theorem ([44] p. 279) that a nonconstant meromorphic function on $\mathbb{C}$ can have at most four totally ramified values. It follows that $g$ is constant, a contradiction.

As a corollary, we have the following sharpening of Schwick's extension [61] of a theorem of Royden [56].

Theorem. Let $\mathcal{F}$ be a family of functions meromorphic on $D \subset \mathbb{C}$ with the property that for each compact set $K \subset D$ there is a function $h_{K}:[0, \infty] \rightarrow[0, \infty]$, which is finite somewhere on $(0, \infty)$, such that

$$
\left|f^{\prime}(z)\right| \leq h_{K}(|f(z)|)
$$

for all $f \in \mathcal{F}$ and $z \in K$. Then $\mathcal{F}$ is normal on $D$.

Proof. Fix $K \subset D$ and take $x_{0}>0$ such that $h_{K}\left(x_{0}\right)<\infty$. Putting $M(K)=$ $h_{K}\left(x_{0}\right)$ and $E(K)=\left\{w \in \mathbb{C}:|w|=x_{0}\right\}$, we have, for each $f \in \mathcal{F}$,

$$
f^{\#}(z) \leq\left|f^{\prime}(z)\right| \leq h_{K}(|f(z)|)=h_{K}\left(x_{0}\right)=M(K)
$$

whenever $z \in K$ and $f(z) \in E(K)$. By the previous theorem, $\mathcal{F}$ is normal on $D$.

This last result yields the normality of the family of solutions (on some common domain) of a differential equation of the form $f^{\prime}=F(f)$, where $|F(w)| \leq h(|w|)$ for 
some function $h$ as above. For example, the solutions to $w^{\prime}=e^{-w^{2}} /(w+1)$ defined on a common domain form a normal family there since

$$
\left|\frac{e^{-w^{2}}}{w+1}\right| \leq \frac{e^{|w|^{2}}}{|| w|-1|} .
$$

In the next section, we shall encounter another application to solutions of first-order differential equations.

5. According to a theorem of A.A. Gol'dberg [26], a meromorphic function on the plane which satisfies a first order algebraic differential equation must have finite order. Recently, Bergweiler [5] observed that the Lemma can be exploited to yield a short proof of a significantly more general result, due to Barsegian [4].

Let $f$ be a meromorphic function on $\mathbb{C}$. For an $m$-tuple $r=\left(r_{1}, r_{2}, \ldots, r_{m}\right)$ of nonnegative integers, define

$$
M_{r}[f](z)=f^{\prime}(z)^{r_{1}} f^{\prime \prime}(z)^{r_{2}} \cdots f^{(m)}(z)^{r_{m}}
$$

and set $M_{(0)}[f](z) \equiv 1$. A differential polynomial $P[f]$ is an expression of the form

$$
P[f](z)=\sum_{r \in I} a_{r}(z, f(z)) M_{r}[f](z),
$$

where the coefficients $a_{r}$ are rational in both variables and the index set is finite. Define the weight of $P[f]$ by

$$
w(P)=\max _{r \in I} w(r),
$$

where $w(r)=r_{1}+2 r_{2}+\cdots+m r_{m}$.

Theorem. A meromorphic function $f$ on $\mathbb{C}$ which satisfies a differential equation of the form $\left(f^{\prime}\right)^{n}=P[f]$, where $P[f]$ is a differential polynomial such that $n>$ $w(P)$, has finite order.

Proof. Assume, to the contrary, that $f$ has infinite order. From the definition of order (cf. $\S 2$ ), one sees easily that there exists a sequence $\left\{w_{k}\right\}$, tending to infinity, such that

$$
\frac{\log f^{\#}\left(w_{k}\right)}{\log \left|w_{k}\right|} \rightarrow \infty
$$

Write $f_{k}(z)=f\left(z+w_{k}\right)$ and consider the family $\mathcal{F}=\left\{f_{k}\right\}$ on $\Delta$. Since $f_{k}^{\#}(0)=$ $f^{\#}\left(w_{k}\right) \rightarrow \infty, \mathcal{F}$ is not normal on $\Delta$. So, by the Lemma, there exist $z_{k} \in \Delta$, $\rho_{k} \rightarrow 0+$, and a nonconstant meromorphic function $g$ on $\mathbb{C}$ such that

$$
f\left(w_{k}+z_{k}+\rho_{k} \zeta\right)=f_{k}\left(z_{k}+\rho_{k} \zeta\right)=g_{k}(\zeta) \rightarrow g(\zeta)
$$

Writing $\tau_{k}=w_{k}+z_{k}$, we see from the proof of the Lemma (cf. $\left.\S 2\right)$ that one may take $\rho_{k}=1 / f_{k}^{\#}\left(z_{k}\right)=1 / f^{\#}\left(\tau_{k}\right)$ and $f^{\#}\left(\tau_{k}\right)=f_{k}^{\#}\left(z_{k}\right) \geq f_{k}^{\#}(0)=f^{\#}\left(w_{k}\right)$. This implies, via (4), that $\tau_{k}^{\ell} \rho_{k} \rightarrow 0$ as $k \rightarrow \infty$ for each fixed $\ell$. Substituting $\tau_{k}+\rho_{k} \zeta$ for $z$ into the differential equation, we have

$$
\rho_{k}^{-n} g_{k}^{\prime}(\zeta)^{n}=\sum_{r \in I} a_{r}\left(\tau_{k}+\rho_{k} \zeta, g_{k}(\zeta)\right) \rho_{k}^{-w(r)} M_{r}\left[g_{k}\right](\zeta)
$$

Multiply both sides by $\rho_{k}^{n}$ and let $k \rightarrow \infty$; the left hand side clearly tends to $g^{\prime}(\zeta)^{n}$, while the right hand side vanishes in the limit since $n-w(r) \geq 1$ for each 
$r \in I$ and $\tau_{k}^{\ell} \rho_{k} \rightarrow 0$ for each fixed $\ell$. Thus $g^{\prime}(\zeta)^{n} \equiv 0$, a contradiction since $g$ is nonconstant.

Taking somewhat greater care in the organization of the argument, Frank and Wang [23] have sharpened this result and obtained an explicit bound for the order of $f$ in terms of the differential equation satisfied by $f$. An example of an equation of the form $\left(f^{\prime}\right)^{n}=P[f]$ with solutions of infinite order is

$$
\left(f^{\prime}\right)^{2}=f f^{\prime \prime \prime}-f f^{\prime \prime}-f^{\prime} f^{\prime \prime},
$$

which is satisfied by $f(z)=e^{e^{z}}$. In this case, $n=2<3=w(P)$.

6. We have already alluded to the historically important role played by normal family techniques in the development of complex dynamics. Even today, judicious application of the Lemma can result in significant simplifications. We illustrate this point with a streamlined proof of one of the principal results of the classical theory.

Let $f$ be a rational function of degree $d \geq 2$ (or an entire function) and consider the collection of iterates $f_{n}, n \in \mathbb{N}$, where $f_{1}=f$ and $f_{n}=f \circ f_{n-1}$. A point $z$ is called periodic if $f_{n}(z)=z$ for some $n \in \mathbb{N}$; it is repelling if $\left|f_{n}^{\prime}(z)\right|>1$. The Julia set $J(f)$ is the complement of the maximal open set on which $\left\{f_{n}\right\}$ is normal. It is well-known, and easy to prove ([10] pp. 56-57), that $J(f)$ is a nonempty perfect set and that $J(f)=J\left(f_{m}\right)$ for each $m \in \mathbb{N}$. We have the following result of Fatou and Julia.

Theorem. Let $f$ be a rational function of degree $d \geq 2$. Then $J(f)$ is the closure of the repelling periodic points.

Proof. (Schwick [62]) We may assume that $d \geq 5$; otherwise, consider $f_{3}$ in place of $f$. Let $A$ be the finite set in $\widehat{\mathbb{C}}$ consisting of $\infty, f(\infty)$, and all critical values of $f$ (i.e., values of $f$ at points where $f^{\prime}$ vanishes). We shall prove that the repelling periodic points are dense in $J(f) \backslash A$; this is sufficient to prove the theorem, as $J(f)$ is a perfect set.

For $w_{0} \in J(f) \backslash A$, apply the Lemma (with $\alpha=0$ ) to the family $\mathcal{F}=\left\{f_{n}\right\}$. We obtain an increasing sequence of positive integers $\left\{n_{k}\right\}$ and $z_{k} \rightarrow w_{0}, \rho_{k} \rightarrow$ $0+$ such that $f_{n_{k}}\left(z_{k}+\rho_{k} \zeta\right)=g_{k}(\zeta) \rightarrow g(\zeta), \chi$-uniformly on compacta, where $g$ is a nonconstant meromorphic function on $\mathbb{C}$. By the choice of $w_{0}$, the equation $f(z)=w_{0}$ has at least five simple roots $z_{1}, \ldots, z_{5} \in \mathbb{C}$. Since $g$ can have at most four totally ramified values, for at least one of the $z_{j}$, say $z_{1}$, there exists $\zeta_{0} \in \mathbb{C}$ with $g\left(\zeta_{0}\right)=z_{1}$ and $g^{\prime}\left(\zeta_{0}\right) \neq 0$.

Consider now

$$
f_{n_{k}+1}\left(z_{k}+\rho_{k} \zeta\right)-\left(z_{k}+\rho_{k} \zeta\right) \rightarrow(f \circ g)(\zeta)-w_{0} .
$$

The right hand side is nonconstant and vanishes for $\zeta=\zeta_{0}$. It follows by Hurwitz's Theorem that, for $k$ sufficiently large, $f_{n_{k}+1}\left(z_{k}+\rho_{k} \zeta\right)=z_{k}+\rho_{k} \zeta$ has a solution $\zeta_{k}$ with $\zeta_{k} \rightarrow \zeta_{0}$. Thus $z_{k}+\rho_{k} \zeta_{k}$ is a fixed point of $f_{n_{k}+1}$, i.e., a periodic point of $f$, and $z_{k}+\rho_{k} \zeta_{k} \rightarrow w_{0}$. It is repelling for large $k$ since $\rho_{k} f_{n_{k}+1}^{\prime}\left(z_{k}+\rho_{k} \zeta_{k}\right)=$ $\left(f \circ g_{k}\right)^{\prime}\left(\zeta_{k}\right) \rightarrow(f \circ g)^{\prime}\left(\zeta_{0}\right)=f^{\prime}\left(g\left(\zeta_{0}\right)\right) g^{\prime}\left(\zeta_{0}\right)=f^{\prime}\left(z_{1}\right) g^{\prime}\left(\zeta_{0}\right) \neq 0$.

With minor modifications, the argument above also establishes

Baker's Theorem. [2] The Julia set of a nonlinear entire function is the closure of the repelling period points. 
Indeed, one may assume that $f$ is transcendental. It follows from Nevanlinna's Second Fundamental Theorem that there exists a (possibly empty) set $A \subset \mathbb{C}$ consisting of at most two points such that for $w \notin A$ the equation $f^{\prime}(z)=w$ has infinitely many simple roots. (Specifically, one takes $A=\{w \in \mathbb{C}: \Theta(w) \geq 1 / 2\}$; cf. [44] p. 280.) The proof then proceeds exactly as before. For the extension of this argument to meromorphic functions (and beyond), see [7].

Baker's original proof made essential use of Ahlfors' Five Islands Theorem, one of the deepest results in all of function theory. The proof given above, depending only on elementary reasoning and a standard result of Nevanlinna Theory, thus represents a significant simplification. A proof that avoids Nevanlinna Theory altogether is in [3].

7. Marty's Theorem provides a complete and satisfying answer to the question of when a family of functions is normal. Unfortunately, in practice it is almost useless, as verification of the condition (1) in cases when normality is not already evident is generally extremely difficult. In many cases, the results in $\S 4$ provide an effective substitute for Marty's Theorem. Long before these results had been obtained, however, the search for more useful sufficient conditions for normality had given rise to the following heuristic principle: "A family of holomorphic (meromorphic) functions which have a property $P$ in common in a domain $D$ is (apt to be) a normal family in $D$ if $P$ cannot be possessed by nonconstant entire (meromorphic) functions in the finite plane" [29] p. 250. This principle is often attributed to André Bloch; however, I have been unable to find any mention of it in his published writings.

In the examples which follow we shall make repeated use of the family of functions $\mathcal{F}=\{n z: n=1,2,3, \ldots\}$, which fails to be normal on the unit disc $\Delta$. Indeed, set $f_{n}(z)=n z$ and let $K$ be any compact subset of $\Delta$ which contains the origin and at least one other point $z_{1}$. No subsequence of $\left\{f_{n}\right\}$ can converge uniformly on $K$ (since $f_{n}\left(z_{1}\right) \rightarrow \infty$ ), nor can any subsequence of $\left\{f_{n}\right\}$ diverge to infinity (as $f_{n}(0)=0$ for all $n$ ).

Examples. 1. Say $f$ has property $P$ on $D$ if $|f(z)| \leq 17$ for $z \in D$. By Liouville's Theorem, any entire function with $P$ is constant. That a family of (holomorphic) functions having $P$ on a domain $D$ is normal follows at once from Montel's Theorem.

2. Say $f$ has $P$ on $D$ if $f(z) \neq a, b, c$ on $D$, where $a, b, c$ are (distinct) fixed values in $\hat{\mathbb{C}}$. Picard's Little Theorem asserts that a meromorphic function on $\mathbb{C}$ with this property must be constant, and Montel's Theorem states that a family of meromorphic functions having $P$ on a domain $D$ is normal.

3. Say $f$ has $P$ on $D$ if $f$ is univalent on $D$, and $f(z) \neq a, b$ on $D$, where $a$ and $b$ are (distinct) fixed values in $\hat{\mathbb{C}}$. We leave the details to the reader.

The heuristic principle has proved itself extremely effective in the identification of criteria which insure normality. However, it must be used with care. Consider, for example, the property " $f$ is not entire". Obviously, no nonconstant entire function has this property (nor, for that matter, do the constants). On the other hand, it is clear that the property does not imply normality. Indeed, fix an analytic function $g$ having the unit circle as a natural boundary and consider the collection of functions $\mathcal{F}=\left\{g_{n}\right\}$ on $\Delta$, where $g_{n}(z)=n z+g(z)$. Clearly, no function in $\mathcal{F}$ is entire; equally clearly, $\mathcal{F}$ is not a normal family.

If the (counter)example of the previous paragraph seems frivolous (and I do not think it is), more serious examples are near at hand. For example, the property 
" $f$ is bounded" forces an entire function to be constant; however, every function in our paradigm non-normal family $\{n z\}$ has this property on $\Delta$. A similar comment applies to the property " $f$ omits three values". Examples like these point up the need for a rigorous version of the heuristic principle. In his retiring presidential address to the Association for Symbolic Logic [54], Abraham Robinson listed this as one of twelve problems worthy of attention of logicians (and, by extension, of mathematicians in general).

It turns out to be possible to answer Robinson's question in a stronger form than he had expected and without recourse to nonstandard analysis (which, naturally enough, he had hoped might provide the key to the solution). However, before turning to this, we need to dispose of the question: what is a property? Answer: a set (viz., the set of all objects having the property!). As we shall be concerned with properties of functions on domains in the plane, it will be convenient to display the domain of the function explicitly together with the function. Thus, following Robinson, we write $\langle f, D\rangle$ to denote the function $f$ defined on the domain $D \subset \mathbb{C}$; and we distinguish between the functions $\langle f, D\rangle$ and $\left\langle f, D^{\prime}\right\rangle$ if $D \neq D^{\prime}$. If the function $f$ has property $P$ on $D$, we write $\langle f, D\rangle \in P$. It is now possible to state the

Theorem. [68] (cf. [69]) Let $P$ be a property of meromorphic (holomorphic) functions which satisfies the following three conditions:

(i) If $\langle f, D\rangle \in P$ and $D^{\prime} \subset D$, then $\left\langle f, D^{\prime}\right\rangle \in P$.

(ii) If $\langle f, D\rangle \in P$ and $\varphi(z)=\rho z+b$ is nonconstant, then $\left\langle f \circ \varphi, \varphi^{-1}(D)\right\rangle \in P$.

(iii) Let $\left\langle f_{n}, D_{n}\right\rangle \in P$, where $D_{1} \subset D_{2} \subset D_{3} \subset \ldots$ and $\bigcup_{n=1}^{\infty} D_{n}=\mathbb{C}$. If $f_{n} \rightarrow f$ $\chi$-uniformly on compact subsets of $\mathbb{C}$, then $\langle f, \mathbb{C}\rangle \in P$.

Suppose $(a)\langle f, \mathbb{C}\rangle \in P$ only if $f$ is constant.

Then $\quad$ (b) $\{f:\langle f, D\rangle \in P\}$ is normal on $D$ for each $D \subset \mathbb{C}$.

Conversely, if (i) and (ii) hold, then (b) implies (a).

This result is a more or less immediate consequence of the Lemma, with $\alpha=0$. It provides a highly satisfactory explication of the heuristic principle so far as properties formulated in terms of the values taken on or omitted by functions is concerned. In such cases, conditions (i) and (ii) will generally be satisfied trivially, while (iii) follows more or less routinely from Hurwitz's Theorem.

Actually, much stronger versions of the Theorem hold. For instance, in (a) one can assume that the function $f$ has order $\leq 2$ (or is of exponential type, in case $f$ is entire). This follows from the fact that the limit function $g$ in the Lemma can be chosen to be such a function. Similarly, condition (ii) can be replaced [48], [49] by the more general

(ii') There exists $\alpha,-1<\alpha<1$, such that if $\langle f, D\rangle \in P$ and $\varphi(z)=\rho z+b$ is nonconstant, then $\left\langle\rho^{\alpha}(f \circ \varphi), \varphi^{-1}(D)\right\rangle \in P$.

This condition is particularly well adapted for dealing with properties formulated in terms of values omitted by derivatives of a function. In the discussion below, we shall restrict ourselves to applications of the Theorem as stated in its pristine form above. 
Examples. (continued)

4. Fix $\varepsilon$ (small) and let $\langle f, D\rangle \in P$ if $f(z) \neq a, b, c$ on $D$, where now $a, b, c \in \hat{\mathbb{C}}$ are allowed to vary with $f$ but $\chi(a, b) \chi(b, c) \chi(c, a) \geq \varepsilon$. By Picard's Little Theorem, (a) holds; hence we obtain a sharpening of the classical version of Montel's Theorem due to Carathéodory (cf. [59] pp. 104-105).

5. Fix $a, b, c \in \hat{\mathbb{C}}$ (distinct) and natural numbers $\ell, m, n$ such that $1 / \ell+1 / m+$ $1 / n<1$. Let $\langle f, D\rangle \in P$ if all $a$-points of $f$ in $D$ have multiplicity at least $\ell$, all $b$-points multiplicity at least $m$, and all $c$-points multiplicity at least $n$. It is an easy consequence of Nevanlinna's Second Main Theorem that if $\langle f, \mathbb{C}\rangle \in P$, then $f$ must be constant. Thus (a) holds, and we obtain a generalization of a result of Montel ([42] pp. 125-126) due to Drasin ([18] pp. 238-239).

6. Say $\langle f, D\rangle \in P$ if $f=g^{\prime}$, where $g$ is (analytic and) univalent on $D$ or $f \equiv 0$ on $D$. (This last possibility is required if (iii) is to hold, as the limit of univalent functions could be constant.) Since the only univalent entire functions are linear, it is clear that $\langle f, \mathbb{C}\rangle \in P$ implies $f$ is constant. Thus the (non-normalized) family of derivatives of all univalent functions on $D \subset \mathbb{C}$ is normal on $D$. By way of contrast, the family of all univalent functions on a domain is not a normal family (consider $\{n z\}$ on $\Delta$ ), nor is the collection of second derivatives of univalent functions (cf. Example 9 below).

One attractive aspect of the Theorem is that it explains the failure of the heuristic principle in those cases where it does not give correct results.

Examples. (continued)

7. Say $\langle f, D\rangle \in P$ if $f$ is bounded on $D$. Clearly (i), (ii), and (a) hold in this case; however, as we have seen, (b) does not follow. This is because (iii) does not obtain. Indeed, fix any nonconstant entire function $f$ and let $D_{n}=\{|z|<n\}$. Then $\left\langle f, D_{n}\right\rangle \in P$ for each $n$, but clearly $\langle f, \mathbb{C}\rangle \notin P$. A similar discussion applies to the properties " $f$ omits 3 (distinct) values on $D$ " and " $f$ is not entire" (i.e., $\langle f, D\rangle \in P \Leftrightarrow D \neq \mathbb{C})$.

8. Say $\langle f, D\rangle \in P$ if $f$ is analytic on $D$ and satisfies $|f(z)| \leq\left|f^{\prime}(z)\right|$ and $0 \in f(D)$. Suppose $\langle f, \mathbb{C}\rangle \in P$. Then $|f(z)| \leq\left|f^{\prime}(z)\right|$ on $\mathbb{C}$, so $f / f^{\prime}$ is constant, and $(\log f)^{\prime}=f^{\prime} / f$ is also. Hence $f(z)=K e^{a z}$ and, since $0 \in f(D), K=0$. Thus the only entire function having $P$ is $f(z) \equiv 0$. That $P$ does not force normality is evident from the family $\{n z\}$ on $\Delta$. In this example, $P$ fails to satisfy any of the conditions (i), (ii), and (iii).

9. Define $\langle f, D\rangle \in P$ if $f=g^{\prime \prime}$, where $g$ is analytic and univalent on $D$. The only entire function with this property is $f(z) \equiv 0$. Setting $g_{n}(z)=n\left(z+z^{2} / 10+z^{3} / 10\right)$, we have $\operatorname{Re} g_{n}^{\prime}(z)>0$ on $\Delta$, so $g_{n}$ is univalent there. Since $f_{n}(z)=g_{n}^{\prime \prime}(z)=$ $n(1 / 5+3 z / 5)$ vanishes at $z=-1 / 3$ for each $n,\left\{f_{n}\right\}$ does not form a normal family on $\Delta$. Clearly (i) and (ii) hold, so it must be (iii) that fails. Verifying this is an amusing exercise, which we leave to the interested reader.

10. Say $\langle f, D\rangle \in P$ if $f$ is analytic on $D$ and $f^{\prime}(z) \neq-1, f^{\prime}(z) \neq-2, f^{\prime}(z) \neq f(z)$ for $z \in D$ or $f \equiv 0$ on $D$. Suppose $\langle f, \mathbb{C}\rangle \in P$; then $f^{\prime}$ is entire, hence constant (since $f^{\prime} \neq-1,-2$ on $\left.\mathbb{C}\right)$. Thus $f(z)=a z+b$. But then $f(z)-f^{\prime}(z)=a z+(b-a) \neq 0$, so that $a=0$ and $f$ is constant. However, $\{n z\}$ has $P$ on $\Delta$. Here it is condition (ii) that fails. It is obvious that (i) holds. To verify (iii), suppose $f_{n} \rightarrow f$ uniformly on compacta, where $f_{n}^{\prime} \neq-1,-2$ and $f_{n}^{\prime}-f_{n} \neq 0$ on $D_{n}$. Then, by Hurwitz's 
Theorem, $f$ must satisfy

A $\quad B$

$$
\begin{array}{lllll}
\text { 1. } & f^{\prime} \neq-1 & \text { or } & f^{\prime} \equiv-1 & \text { (i.e., } f(z)=-z+b) \\
\text { 2. } & f^{\prime} \neq-2 & \text { or } & f^{\prime} \equiv-2 & \text { (i.e., } f(z)=-2 z+c) \\
\text { 3. } & f^{\prime}-f \neq 0 & \text { or } & f^{\prime}-f \equiv 0 & \text { (i.e., } \left.f(z)=K e^{z}\right) .
\end{array}
$$

$1 B$ and $2 B$ contradict 3 , and $3 B$ contradicts 1 and 2 unless $K=0$. Thus $\langle f, \mathbb{C}\rangle \in P$.

These last two examples of the failure of the heuristic principle are due to Rubel [57]. They show clearly that neither (ii) nor (iii) may be dispensed with in the formulation of the Theorem.

8. In his little problem book [28], Hayman posed a number of open problems on normal families. Hayman's questions on normal families are all of similar shape: in each case, a property involving the values of a function and its derivatives is known to imply that an entire or globally defined meromorphic function must be constant. Does the same property imply normality for a family of holomorphic or meromorphic functions? (Of course, this is just a special case of the "Bloch Principle" discussed above. ${ }^{2}$ ) Over the years, most of these questions have been answered (affirmatively); let us indicate how the Lemma can be used to provide simple and uniform solutions to these problems.

We begin with the first, and easiest, of these problems, 5.11 of [28]. Fix $n \geq 1$. It is classical that an entire function which satisfies $f \neq 0, f^{(n)} \neq 1$ must be constant; Hayman [27] proved that this remains true for meromorphic functions on $\mathbb{C}$. That a family of analytic functions on a domain $D$ with this property is normal on $D$ goes back to Miranda [41]. It was considered a great advance when $\mathrm{Gu}$ showed that the family of meromorphic functions on $D$ such that $f \neq 0, f^{(n)} \neq 1$ on $D$ is normal there [33].

The gap of almost half a century that separates Gu's theorem from Miranda's testifies to the formidable technical difficulties involved in extending a result for holomorphic functions to meromorphic functions. Remarkably, the Lemma does not distinguish between these cases. Accordingly, when it can be applied (and when appropriate results for globally defined individual functions are available), it yields normality results for families of meromorphic functions. Let us illustrate this point in the current situation.

Denote the family in question by $\mathcal{F}$ and suppose that $\mathcal{F}$ is not normal. As usual, one may assume $D=\Delta$. Choose $\alpha=-n$ (as we may since $f \neq 0$ for $f \in \mathcal{F}$ ). By the Lemma, there exist $f_{k} \in \mathcal{F}$ and $z_{k}, \rho_{k}$ such that $\rho_{k}^{-n} f_{k}\left(z_{k}+\rho_{k} \zeta\right)=g_{k}(\zeta) \rightarrow g(\zeta)$ locally $\chi$-uniformly, where $g$ is a nonconstant meromorphic function on $\mathbb{C}$. Since $g_{k} \neq 0$ and $g$ is nonconstant, $g \neq 0$ by Hurwitz's Theorem. Also, $f_{k}^{(n)}\left(z_{k}+\rho_{k} \zeta\right)=$ $g_{k}^{(n)}(\zeta) \rightarrow g^{(n)}(\zeta)$; hence, since $f_{k}^{(n)} \neq 1$, either $g^{(n)} \neq 1$ or $g^{(n)} \equiv 1$. The latter is impossible, for then $g$ would be a nonconstant polynomial and $g \neq 0$ would contradict the Fundamental Theorem of Algebra. Thus $g \neq 0, g^{(n)} \neq 1$. But then $g$ is constant by Hayman's theorem, a contradiction.

\footnotetext{
${ }^{2}$ And, in point of fact, many (though not all) of Hayman's problems can be settled by invoking the Theorem of the previous section (in its generalized form) with an appropriate choice of $\alpha$; cf. [70]. Here we prefer the slightly more direct (and more general) approach to these problems via the Lemma.
} 
The above method is easily adapted to handle the extension of Gu's theorem in which the condition $f^{(n)} \neq 1$ is replaced by

$$
h(z) \equiv f^{(n)}(z)+\sum_{j=1}^{n} a_{n-j}(z) f^{(n-j)}(z) \neq 1,
$$

where $a_{0}(z), a_{1}(z), \ldots, a_{n-1}(z)$ are fixed holomorphic functions. For holomorphic functions, this is due to Chuang and is one of the main results in [18]. The reader is invited to try his hand at proving this result for meromorphic functions (which do not vanish) using the Lemma.

Problems 5.12 and 5.13 of [28] deal with the condition $f^{\prime} f^{n} \neq 1$ where $n \geq 1$ is fixed. This condition is known to force an entire function (Hayman [27] for $n \geq 2$, Clunie [16] for $n=1$ ) or a meromorphic function on $\mathbb{C}$ (Hayman [27] for $n \geq 3$, Mues [43] for $n=2$ ) to be constant. The corresponding normality results are due to Yang and Zhang [66] (for analytic functions, $n \geq 2$ ) and [67] (for meromorphic functions, $n \geq 5$ ), Gu [32] (for meromorphic functions, $n=3,4$ ), Oshkin [45] (for analytic functions, $n=1$; cf. Li and Xie [37]), and Pang [48] (for meromorphic functions, $n=2$ ).

Here is a simple proof, based on the Lemma, that the family $\mathcal{F}$ of functions meromorphic on $\Delta$ which satisfy $f^{\prime} f^{n} \neq 1$ ( $n \geq 2$ fixed) is a normal family on $\Delta$. Suppose that $\mathcal{F}$ is not normal. Taking $\alpha=-\frac{\overline{1}}{n+1}$, we may choose $f_{k} \in \mathcal{F}, z_{k} \in \Delta$ and $\rho_{k} \rightarrow 0+$ such that

$$
\rho_{k}^{-\frac{1}{n+1}} f_{k}\left(z_{k}+\rho_{k} \zeta\right)=g_{k}(\zeta) \rightarrow g(\zeta)
$$

locally $\chi$-uniformly, where $g$ is a nonconstant meromorphic function on $\mathbb{C}$. Now

$$
f_{k}^{\prime}\left(z_{k}+\rho_{k} \zeta\right) f_{k}^{n}\left(z_{k}+\rho_{k} \zeta\right)=g_{k}^{\prime}(\zeta) g_{k}^{n}(\zeta) \rightarrow g^{\prime}(\zeta) g^{n}(\zeta)
$$

since $f_{k}^{\prime} f_{k}^{n} \neq 1$, either $g^{\prime} g^{n} \neq 1$ or $g^{\prime} g^{n} \equiv 1$. But if $g^{\prime} g^{n} \equiv 1$, then $g^{n+1}(\zeta)=$ $(n+1) \zeta+C$, an impossibility (since $g$ is single-valued). Thus $g^{\prime} g^{n} \neq 1$. But then $g$ must be constant, a contradiction.

All that prevents the above proof from settling the case $n=1$ is the absence of the corresponding theorem for global meromorphic functions. However, we can actually get by with much less. In fact, Bergweiler and Eremenko [6] have proved that a function of finite order which satisfies $f^{\prime} f \neq 1$ must be constant. Since the limit function $g$ may be taken to have finite order, we see that the above proof establishes that the condition $f^{\prime} f \neq 1$ also implies normality.

Once the normality criterion is established, it is easy to conclude that the only meromorphic functions on $\mathbb{C}$ for which $f^{\prime} f \neq 1$ are constant; this settles problem 1.19 in [28]. Indeed, let $f$ be such a function, and suppose that $f$ is not constant. Then we can find $z_{0} \in \mathbb{C}$ such that $f^{\#}\left(z_{0}\right) \neq 0$. Let $f_{k}(z)=k^{-1 / 2} f\left(z_{0}+k z\right)$ for $z \in \Delta$. Clearly, $f_{k}^{\prime}(z) f_{k}(z) \neq 1$ on $\Delta$, so that $\mathcal{F}=\left\{f_{k}\right\}$ is a normal family. By Marty's Theorem, there exists $C>0$ such that $f_{k}^{\#}(0) \leq C$ for all $k$. But $f_{k}^{\#}(0) \geq \sqrt{k} f^{\#}\left(z_{0}\right)$, which tends to infinity, a contradiction.

The results discussed above concerning the condition $f^{\prime} f \neq 1$ are due, independently and simultaneously, to Bergweiler and Eremenko [6], Chen and Fang [12], and Zalcman [71].

It seems worth commenting on the "philosophy" of the proof of this result, which flies in the face of the conventional wisdom concerning proving theorems on entire and meromorphic functions. It has generally been felt that proofs involving special 
properties of functions of finite order should be avoided whenever possible and, where order considerations are unavoidable, that the lower order (obtained by replacing lim sup in (2) by lim inf) should be used in preference to the order. While this has often led to proofs of stronger results than would otherwise have been obtained, the results of this section suggest adopting a less doctrinaire stance. Indeed, what is remarkable about the solution for the case $n=1$ is that a result established initially only for functions of finite order is shown by very general considerations to be valid for arbitrary meromorphic functions. It is to be expected that this same approach will prove successful in dealing with a variety of other problems. For a first step in this direction, see [11], [50].

For complete solutions of all of Hayman's problems on normal families via the Lemma, see [72].

9. In this report, we have focused on applications to one-dimensional complex analysis. It turns out that the ideas involved in the Lemma have much wider applicability. In this final section we survey briefly three such directions.

a. Quasimeromorphic mappings. The theory of quasiregular and quasimeromorphic mappings [53] is a generalization to $\mathbb{R}^{n}$ of the theory of holomorphic and meromorphic functions on the plane, bearing much the same relation to that theory that the theory of quasiconformal mappings in space [63] bears classical conformal mapping. Ruth Miniowitz [40] has generalized our Lemma to families of $K$-quasimeromorphic mappings on the unit ball $B^{n}$. As a consequence, she obtains analogues of Bloch's Principle, Montel's Theorem, and the Big Picard Theorem for quasimeromorphic mappings. It should be mentioned that these last two results depend on Rickman's extension of the Little Picard Theorem to quasiregular functions [52]. It would be interesting to adapt the argument of $\S 3$ to provide an independent proof of this important result, but I have not seen how to do it. Quite recently, Miniowitz's result has been used by Eremenko [19] to extend the classical covering theorem of Bloch ([29] pp. 385-388) to quasimeromorphic mappings on $\mathbb{R}^{n}$.

b. Hyperbolicity. Recall that a complex manifold $M$ is hyperbolic if the Kobayashi pseudodistance $d_{M}(p, q)$ is actually a metric [31], Chapter 4 . It is easy to see that if $M$ contains a complex line (i.e., if there exists a nonconstant holomorphic mapping $g: \mathbb{C} \rightarrow M)$, then $M$ is not hyperbolic, since then $d_{M}(g(z), g(w))=0$ for all $z, w \in \mathbb{C}$. The converse fails, as one sees by taking

$$
M=\{(z, w):|z|<1, \quad|z w|<1 \quad \text { and } \quad|w|<1 \quad \text { if } \quad z=0\} .
$$

(This example is due to D. Pelles (Eisenman) and L. Taylor [31] p. 130.) However, for compact manifolds one has

Brody's Theorem. [9] (cf. [34] p. 68) A compact complex manifold is hyperbolic if and only if it contains no complex lines.

The ideas used in the proof of this result are closely related to the Lemma; indeed, as pointed out by $\mathrm{Wu}$ [64] p. 95, Brody's Theorem follows at once from the argument used to prove the Lemma. For further developments in this direction, see [34] Chapter 3.

c. Minimal surfaces. Let $M$ be a complete minimal surface in $\mathbb{R}^{3}$. The Gauss map assigns to each point $p \in M$ the unit normal to $M$ at $p$, regarded as a point in the unit sphere $S^{2} \subset \mathbb{R}^{3}$. In 1959, Osserman [46] showed that the Gauss map of a complete minimal surface in $\mathbb{R}^{3}$ must be dense in $S^{2}$ unless $M$ is a plane (in which 
case it is a constant), thereby initiating a sequence of developments (described in [47]; cf. [25]) which culminated in

Fujimoto's Theorem. [24] The Gauss map of a complete minimal surface $M$ in $\mathbb{R}^{3}$ omits at most four points unless $M$ is a plane.

This is optimal, as it is not difficult to construct minimal surfaces whose Gauss maps omit any four (or fewer) prescribed points in $S^{2}$. In a very interesting paper [55], Antonio Ros has shown how the ideas around the Lemma can be adapted to deal with minimal surfaces and, in particular, to simplify the proof of Fujimoto's Theorem. This appears to be a most promising direction of future research.

10. For a systematic account of the theory of normal families, the reader is directed to [42], [59], and [15]. The monograph [59], in particular, provides an admirable introit to the modern theory.

\section{REFERENCES}

1. Gerardo Aladro and Steven G. Krantz, A criterion for normality in $\mathbb{C}^{n}$, J. Math. Anal. Appl. 161 (1991), 1-8. MR 92j:32004

2. I.N. Baker, Repulsive fixpoints of entire functions, Math. Z. 104 (1968), 252-256. MR 37:1599

3. Detlef Bargmann, Simple proofs of some fundamental properties of the Julia set, preprint.

4. G. Barsegian, Geometrical theory of meromorphic functions, manuscript.

5. Walter Bergweiler, On a theorem of Gol'dberg concerning meromorphic solutions of algebraic differential equations, Complex Variables Theory Appl. (to appear).

6. Walter Bergweiler and Alexandre Eremenko, On the singularities of the inverse to a meromorphic function of finite order, Rev. Mat. Iberoamericana 11 (1995), 355-373. MR 96h:30055

7. Andreas Bolsch, Repulsive periodic points of meromorphic functions, Complex Variables Theory Appl. 31 (1996), 75-79. MR 98c:30033

8. Mario Bonk and Alexandre Eremenko, Schlicht regions for entire and meromorphic functions, preprint.

9. Robert Brody, Compact manifolds and hyperbolicity, Trans. Amer. Math. Soc. 235 (1978), 213-219. MR 57:10010

10. Lennart Carleson and Theodore W. Gamelin, Complex Dynamics, Springer, 1993. MR 94h:30033

11. Chen Huaihui, Yosida functions and Picard values of integral functions and their derivatives, Bull. Austral. Math. Soc. 54 (1996), 373-381. MR 97k:30035

12. Chen Huaihui and Fang Mingliang, On the value distribution of $f^{n} f^{\prime}$, Sci. China Ser. A $\mathbf{3 8}$ (1995), 789-798. MR 97a:30035

13. Chen Huaihui and Gu Yongxing, An improvement of Marty's criterion and its applications, Sci. China Ser. A 36 (1993), 674-681. MR 94j:30031

14. Huai-hui Chen and Xin-hou Hua, Normality criterion and singular directions, Proceedings of the Conference on Complex Analysis, (Tianjin, 1992), International Press, 1994, pp. 34-40. MR 96d:30039

15. Chi-Tai Chuang, Normal Families of Meromorphic Functions, World Scientific, 1993. MR 95d:30065

16. J. Clunie, On a result of Hayman, J. London Math. Soc. 42 (1967), 389-392. MR 35:5618

17. J. Clunie and W.K. Hayman, The spherical derivative of integral and meromorphic functions, Comment. Math. Helv 40 (1966), 117-148. MR 33:282

18. David Drasin, Normal families and the Nevanlinna theory, Acta Math. 122 (1969), 231-263. MR 40:2835

19. Alexandre Eremenko, Bloch radius, normal families, and quasiregular mappings, preprint.

20. Normal holomorphic curves from parabolic regions to projective spaces, preprint.

21. Jan-Erik Fornaess, Dynamics in Several Complex Variables, CBMS 87, Amer. Math. Soc., Providence, 1996. MR 96j:32033

22. Günter Frank and Wilhelm Schwick, A counterexample to the generalized Bloch principle, New Zealand J. Math. 23 (1994), 121-123. MR 95m:30044 
23. Günter Frank and Yufei Wang, On the meromorphic solutions of algebraic differential equations, preprint.

24. Hirotaka Fujimoto, On the number of exceptional values of the Gauss map of minimal surfaces, J. Math. Soc. Japan 40 (1988), 237-249. MR 89b:53013

25. - Value Distribution Theory of the Gauss Map of Minimal Surfaces in $R^{m}$, Vieweg, Braunschweig, 1993. MR 95d:32029

26. A.A. Gol'dberg, On single-valued solutions of first-order differential equations, Ukrain. Math. Zh. 8 (1956), 254-261.

27. W.K. Hayman, Picard values of meromorphic functions and their derivatives, Ann. of Math. (2) 70 (1959), 9-42. MR 22:1675

28. _ Research Problems in Function Theory, Athlone Press, London, 1967. MR 36:359

29. Einar Hille, Analytic Function Theory, Vol. 2, Ginn, Boston, 1962. MR 34:1490

30. A. Hinkkanen, Normal families and Ahlfors's five islands theorem, New Zealand J. Math. 22 (1993), 39-41. MR 95b:30049

31. Shoshichi Kobayashi, Hyperbolic Manifolds and Holomorphic Mappings, Marcel Dekker, New York, 1970. MR 43:3503

32. Ku Yung-hsing, Sur les familles normales de fonctions méromorphes, Sci. Sinica 21 (1978), 431-445. MR 80a:30033

33. $\mathrm{Ku}$ Yongxing, Un critère de normalité des familles de fonctions méromorphes, Sci. Sinica Special Issue 1 (1979), 267-274 (Chinese).

34. Serge Lang, Introduction to Complex Hyperbolic Spaces, Springer, 1987. MR 88f:32065

35. Peter Lappan, A criterion for a meromorphic function to be normal, Comment. Math. Helv. 49 (1974), 492-495. MR 52:755

36. _ A uniform approach to normal families, Rev. Roumaine Math. Pures Appl. 39 (1994), 691-702. MR 96b:30077

37. Li Song-ying and Xie Hui-chun, On normal families of meromorphic functions, Acta Math. Sinica 29 (1986), 468-476 (Chinese). MR 88f:30048

38. F. Marty, Recherches sur le répartition des valeurs d'une fonction méromorphe, Ann. Fac. Sci. Univ. Toulouse (3) 23 (1931), 183-261.

39. David Minda, Yosida functions, Lectures on Complex Analysis (Xian, 1987) (Chi-Tai Chuang, ed.), World Scientific Pub. Co, Singapore, 1988, pp. 197-213. MR 90d:30097

40. Ruth Miniowitz, Normal families of quasimeromorphic mappings, Proc. Amer. Math. Soc. 84 (1982), 35-43. MR 83c:30026

41. Carlo Miranda, Sur un nouveau critère de normalité pour les familles de fonctions holomorphes, Bull. Soc. Math. France 63 (1935), 185-196.

42. Paul Montel, Leçons sur les familles normales des fonctions analytiques et leurs applications, Gauthier-Villars, Paris, 1927.

43. Erwin Mues, Über ein Problem von Hayman, Math. Z. 164 (1979), 239-259. MR 80d:30026

44. Rolf Nevanlinna, Analytic Functions, Springer, 1970. MR 43:5003

45. I.B. Oshkin, On a test for the normality of families of holomorphic functions, Uspehi Mat. Nauk 37 (2) (1982), 221-222; Russian Math. Surveys 37 (2) (1982), 237-238. MR 83f:30026

46. Robert Osserman, Proof of a conjecture of Nirenberg, Comm. Pure Appl. Math. 12 (1959), 229-232. MR 21:4436

47. _ Minimal surfaces in $\mathbb{R}^{3}$, in Global Differential Geometry (S.S. Chern, ed.), Math. Assoc. Amer., 1989, pp. 73-98. MR 90g:53012

48. Pang Xue-cheng, Bloch's principle and normal criterion, Sci. China Ser. A 32 (1989), 782-791. MR 91i:30031

49. , On normal criterion of meromorphic functions, Sci. China Ser. A 33 (1990), 521-527. MR 92b:30041

50. Pang Xue-cheng and Lawrence Zalcman, On theorems of Hayman and Clunie, New Zealand J. Math. (to appear).

51. Normal families and shared values, preprint.

52. Seppo Rickman, On the number of omitted values of entire quasiregular mappings, J. Analyse Math. 37 (1980), 100-117. MR 81m:30030

53. _ Quasiregular Mappings, Springer, 1993. MR 95g:30026

54. Abraham Robinson, Metamathematical problems, J. Symbolic Logic 38 (1973), 500-516. MR 49:2240

55. Antonio Ros, The Gauss map of minimal surfaces, preprint. 
56. H.L. Royden, A criterion for the normality of a family of meromorphic functions, Ann. Acad. Sci. Fenn. Ser. A. I 10 (1985), 499-500. MR 86i:30040

57. Lee A. Rubel, Four counterexamples to Bloch's principle, Proc. Amer. Math. Soc. 98 (1986), 257-260. MR 87i:30064

58. Stanisław Saks and Antoni Zymund, Analytic Functions, 3rd ed., Elsevier, 1971.

59. Joel L. Schiff, Normal Families, Springer, 1993. MR 94f:30046

60. Wilhelm Schwick, Normality criteria for families of meromorphic functions, J. Analyse Math. 52 (1989), 241-289. MR 90k:30061

61. On a normality criterion of H.L. Royden, New Zealand J. Math. 23 (1994), 91-92. MR 95g:30047

62. _ Repelling periodic points in the Julia set, Bull. London Math. Soc. 29 (1997), 314-316. MR 97m:30029

63. Jussi Väisälä, Lectures on n-Dimensional Quasiconformal Mappings, Lecture Notes in Mathematics 229, Springer, 1971. MR 56:12260

64. H. Wu, Some theorems on projective hyperbolicity, J. Math. Soc. Japan 33 (1981), 79-104. MR 82j:53061

65. Xue Guo-fen and Pang Xue-cheng, A criterion for normality of a family of meromorphic functions, J. East China Norm. Univ. Natur. Sci. Ed. (1988), no. 2, 15-22 (Chinese). MR 90i:30051

66. Yang Le and Chang Kuang-hou, Recherches sur la normalité des familles de fonctions analytiques à des valeurs multiples, I. Un nouveau critère et quelques applications, Sci. Sinica 14 (1965), 1258-1271.

67. __ Recherches sur la normalité des familles de fonctions analytiques à des valeurs multiples, II. Généralisations, Sci. Sinica 15 (1966), 433-453.

68. Lawrence Zalcman, A heuristic principle in complex function theory, Amer. Math. Monthly 82 (1975), 813-817. MR 52:757

69. _ Modern perspectives on classical function theory, Rocky Mountain J. Math. 12 (1982), 75-92. MR 83d:30003

70. - Normal families revisited, Complex Analysis and Related Topics (J.J.O.O. Wiegerinck, ed.), University of Amsterdam, 1993, pp. 149-164.

71. - On some questions of Hayman, unpublished manuscript, 5pp., 1994.

72. N_ New light on normal families, Proceedings of the Ashkelon Workshop on Complex Function Theory (May, 1996) (L. Zalcman, ed.), Bar-Ilan Univ., 1997, pp. 237-245. CMP 98:03

Department of Mathematics and Computer Science, Bar-Ilan University, Ramat-Gan 52900, ISRAEL

E-mail address: zalcman@macs.biu.ac.il 